\title{
Breastfeeding reduces risk of type 2 diabetes mellitus
}

Research
studies are
needed to
elucidate the
biological
mechanisms

The health benefits garnered by women who breastfeed might be greater than previously thought, according to a new study published in JAMA Internal Medicine. The authors report a strong association of breastfeeding duration with a lower incidence of type 2 diabetes mellitus (T2DM).

"Previous studies were limited in their ability to assess early conversion to diabetes mellitus after pregnancy because most women without diabetes entered these studies many years to decades after their pregnancies," explains lead author Erica Gunderson. "In addition, these studies relied entirely on a self-report of diabetes mellitus, without any systematic longitudinal biochemical testing and they did not account for a history of gestational diabetes in women of childbearing age, which is a very strong risk factor for T2DM."
Gunderson and colleagues analysed data from the CARDIA Study - a longitudinal, prospective cohort study conducted in women of childbearing age that measured glucose tolerance and diabetes mellitus medication use, both before and after pregnancy, approximately every 5 years during the 30 -year study follow-up period. In the present study, the authors assessed breastfeeding duration across all births in relation to the incidence of T2DM in women, accounting for pre-pregnancy obesity and metabolic measures, perinatal outcomes, history of gestational diabetes, weight changes and lifestyle behaviours.

The authors report that longer duration of breastfeeding was associated with a marked decrease in the risk of T2DM after accounting for lifestyle behaviours, pre-pregnancy metabolic status, gestational diabetes, and perinatal outcomes. Specifically, $>6$ months of breastfeeding reduced the risk of T2DM by half compared with never breastfeeding.

"Research studies are needed to elucidate the biological mechanisms that could underlie the relationship between breastfeeding duration and lower diabetes mellitus risk," concludes Gunderson. "This is unlikely to be due to obesity because 30-year weight gain did not weaken the association of breastfeeding with diabetes risk in our study."

Alan Morris

ORIGINAL ARTICLE Gunderson, E. P. et al. Lactation duration and progression to diabetes in women across the childbearing years: the 30-year CARDIA study. JAMA Intern. Med. https:// dx.doi.org/10.1001/jamainternmed.2017.7978 (2018) 